\title{
Apelin-13 stimulates angiogenesis by promoting cross-talk between AMP-activated protein kinase and Akt signaling in myocardial microvascular endothelial cells
}

\author{
XIAOMAN YANG ${ }^{*}$, WEI ZHU* ${ }^{*}$ PU ZHANG, KANKAI CHEN, \\ LIJIE ZHAO, JINGBO LI, MENG WEI and MINGYA LIU \\ Division of Cardiology, Shanghai 6th People's Hospital, Shanghai Jiao Tong University School of Medicine, \\ State Key Discipline Division, Shanghai 200233, P.R. China
}

Received June 19, 2013; Accepted February 3, 2014

DOI: $10.3892 / \mathrm{mmr} .2014 .1984$

\begin{abstract}
Currently, there is major interest in the functions of apelin-13, an endogenous ligand for the orphan G-protein coupled receptor APJ, a receptor that closely resembles the angiotensin receptor AT1. In the present study, the role of apelin-13 in angiogenesis and its mechanism as a novel angiogenic factor in myocardial microvascular endothelial cells (MMVECs) was investigated. It was revealed that apelin-13 can promote proliferation, migration and tube formation in MMVECs. In addition, apelin-13 dose dependently stimulated the phosphorylation of AMP-activated protein kinase (AMPK) and endothelial nitric oxide synthase (eNOS) at Thr-172 and Ser-1179, respectively. The treatment with the AMPK (compound C) and protein kinase Akt/protein kinase B (Akt; LY294002) inhibitor significantly suppressed the apelin-13-induced AMPK, Akt and eNOS phosphorylation. They also inhibited the apelin13-stimulated endothelial cell migration and tube formation. Therefore, we hypothesize that apelin-13 promotes angiogenesis through the modulation of AMPK and Akt signaling in MMVECs.
\end{abstract}

Correspondence to: Professor Mingya Liu and Professor Meng Wei, Division of Cardiology, Shanghai 6th People's Hospital, Shanghai Jiao Tong University School of Medicine, State Key Discipline Division, 600 Yishan Road, Shanghai 200233, P.R. China E-mail: 1lmylmy@126.com

Email:mrweei@medmail.com.cn

${ }^{*}$ Contributed equally

Abbreviations: AMPK, AMP-activated protein kinase; eNOS, endothelial nitric oxide synthase; Akt, protein kinase Akt/protein kinase B; MMVECs, myocardial microvascular endothelial cells; VEGF, vascular endothelial growth factor; FBS, fetal bovine serum; GPCR, orphan G-protein-coupled receptor; ERK, extracellular signal-regulated kinase; NO, nitric oxide

Key words: apelin-13, AMP-activated protein kinase, Akt, endothelial nitric oxide synthase, angiogenesis

\section{Introduction}

Apelin is a novel adipocyte-derived factor that binds the orphan G protein-coupled receptor (GPCR) APJ with high affinity (1). Apelin mRNA encodes a 77-amino-acid prepropeptide, which is proteolytically cleaved to yield bioactive peptides of 36,17 and 13 amino acids (aa) in size. Each of these peptides contains the extreme $\mathrm{C}$-terminal region of the precursor protein and their bioactivity resides in the C-terminal 13 aa fragment (2). The sequence of the 13 aa apelin peptide is highly conserved among different species, suggesting that its critical function has been evolutionarily conserved (3).

The apelinergic system has a widespread pattern of distribution $(4,5)$. However, the majority of studies regarding the function of apelin/APJ are on the cardiovascular system, due to its similarity to the angiotensin receptor. In the cardiovascular system, apelin reduces blood pressure and modulates the contractility of cardiac tissue and blood vessels $(6,7,8)$. Apelin signaling is also associated with tumor angiogenesis and vascular regeneration during embryogenesis (9).

AMP-activated protein kinase (AMPK) is a stress-activated protein kinase, which is involved in the regulation of energy and metabolic homeostasis (10). In vascular endothelium, AMPK signaling is required for vascular endothelial growth factor (VEGF)-induced nitric oxide (NO) production, migration and differentiation under conditions of hypoxia (11). Previous studies indicated that apelin-13 is able to regulate glucose and lipid metabolism $(12,13)$ via the activation of AMPK signaling. The disruption of apelin-13 signaling can increase the hypoxia-induced pulmonary hypertension mediated by decreased activation of AMPK and endothelial nitric oxide synthase (eNOS) (14). In addition, as protein kinase Akt/protein kinase B (Akt) signaling participates in vascular homeostasis and angiogenesis $(15,16)$, it can also phosphorylate eNOS (17), resulting in NO production and subsequently leading to the regulation of vasomotor responses. Thus, it was hypothesized that AMPK and Akt signaling may participate in the regulation of angiogenesis stimulated by apelin-13.

The aim of the present study was to investigate the role of apelin-13 in angiogenesis in rat primary myocardial microvascular endothelial cells (MMVECs) and the mechanisms 
involved in it. In addition, compound C, an AMPK inhibitor, and LY294002, an Akt inhibitor were used to investigate whether AMPK and Akt signaling participate in the pro-angiogenic process induced by apelin-13.

\section{Materials and methods}

Materials. Apelin-13 was purchased from GL Biochem Shanghai (Shanghai, China). Dulbecco's modified Eagle's medium(DMEM) and fetal bovine serum(FBS) were purchased from Gibco-BRL (Carlsbad, CA, USA). Phospho-AMPK (Thr-172), pan- $\alpha$-AMPK, phospho-Akt (Ser-473) and phospho-eNOS (Ser-1179) antibodies were purchased from Cell Signaling Technology (Beverly, MA, USA). Akt, eNOS and GAPDH antibodies were purchased from Santa Cruz Biotechnology, Inc. (Santa Cruz, CA, USA). Compound C was purchased from Toronto Research Chemicals Inc. (North York, ON, Canada) and LY294002 from Calbiochem (San Diego, CA, USA). Secondary antibodies were purchased from Beijing Biosynthesis Biotechnology Co., Ltd. (Beijing, China). All antibodies were polyclonal, and sourced from rabbit.

Isolation and identification of MMVECs in rats. Wistar rats (80-100 g) were used for the isolation of primary MMVECs. The animal protocol was approved by the Animal Care Committee of Shanghai Jiao Tong University (Shanghai, China). Briefly, rats were anesthetized with sodium pentobarbital $(60 \mathrm{mg} / \mathrm{kg})$ and heparinized by intraperitoneal injection of sodium heparin (500 U/0.1 kg). Following thoracotomy, the heart was rapidly dislodged and washed in phosphate-buffered saline (PBS). The atria, visible connective tissue, the right ventricle and the epicardial and endocardial surfaces of the left ventricle were carefully removed and the remaining myocardial tissue was washed in PBS with antibiotics (penicillin-streptomycin; 15140-122; Life Technologies, Carlsbad, CA, USA) several times prior to cutting into $1-\mathrm{mm}^{3}$ sections without visible vessels. Myocardial tissues were seeded on culture plates pre-coated with rat tail tendon gelatin and incubated at $37^{\circ} \mathrm{C}$ in a humidified atmosphere of $5 \% \mathrm{CO}_{2}$ for $30 \mathrm{~min}$. The tissues were cultured in DMEM (4500 mg/l; D-glucose) supplemented with $20 \%$ FBS, $50 \mathrm{U} / \mathrm{ml}$ heparin, $100 \mathrm{U} / \mathrm{ml}$ penicillin and $100 \mu \mathrm{g} / \mathrm{ml}$ streptomycin. The tissue sections were discarded after the cells began to grow and the medium was changed at $70 \mathrm{~h}$ intervals. MMVECs were identified by their typical 'cobblestone' appearance and by positive CD31 and CD34 immunostaining. MMVECs at the second passage were used for experiments. The cells were allowed to grow to $80-90 \%$ confluence and were used for further experimental analysis.

Cell proliferation assay. The proliferation rate of MMVECs was determined by the MTT assay. The cells were trypsinized and resuspended in 10\% DMEM. The cells were seeded at $2 \times 10^{3}$ cells per well in 96-well plates and cultured in a humidified $5 \% \mathrm{CO}_{2}$ atmosphere at $37^{\circ} \mathrm{C}$ for $24 \mathrm{~h}$. Drugs (apelin-13, compound C and LY294002) were added to the medium at the indicated times with different concentrations. Following incubation, the cells were washed with PBS and subsequently incubated with MTT at $37^{\circ} \mathrm{C}$ for $4 \mathrm{~h}$. Following incubation, dimethylsulfoxide was added, the cells were incubated for $10 \mathrm{~min}$ and the absorbance at $490 \mathrm{~nm}$ was recorded with a

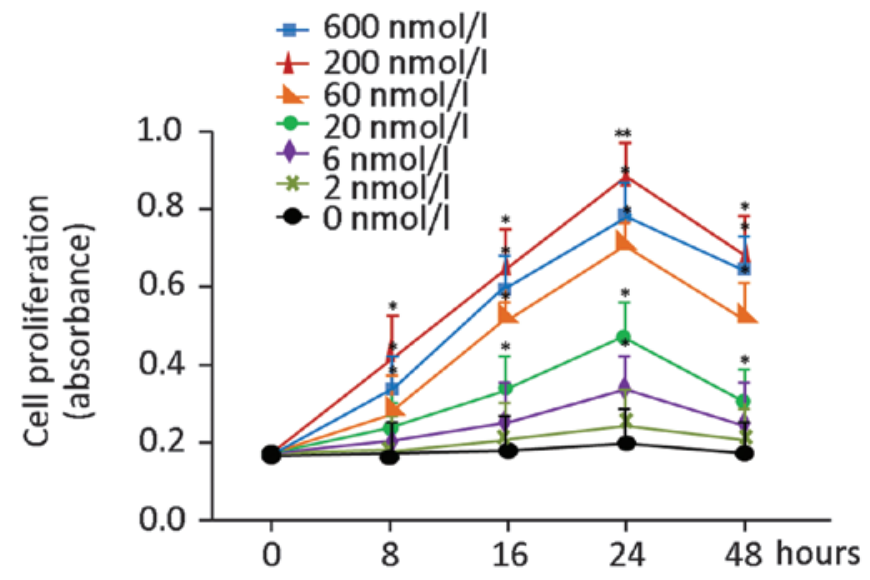

Figure 1. Apelin-13 promotes the proliferation of MMVECs. Proliferation was assessed using the MTT assay. MMVECs were seeded in 96-well dishes in the presence of different concentrations $(0-600 \mathrm{nmol} / 1)$ of apelin-13 for $0,8,16,24$ and $48 \mathrm{~h}$. Data are expressed as the mean \pm standard deviation from three independent experiments. ${ }^{*} \mathrm{P}<0.05,{ }^{* *} \mathrm{P}<0.01$ as compared with the control. MMVECs, myocardial microvascular endothelial cells.

Epoch Microplate spectrophotometer (BioTek Instruments, Inc., Winooski, VT, USA).

\section{Migration assays}

Cell scratch assay. MMVECs were trypsinized and resuspended in 10\% DMEM. The cells were incubated in 6 -well culture dishes in DMEM containing 10\% FBS until $90 \%$ confluency. Following serum starvation for $24 \mathrm{~h}$, a linear wound was made by scratching the bottom of the dish. Following wounding, cells were washed with PBS and incubated in serum-free DMEM. Following treatment, cells were allowed to migrate for $0,8,16$ and $24 \mathrm{~h}$. The wound width was measured in three areas selected at random and images were captured using the Motic AE31 Photometry and Dimensioning microscope (Milton, MA, USA). Migration was quantified by the assessment of the migration distance beyond the reference line using the Motic Image Plus software (Houston, TX, USA).

Boyden chamber assay. The Boyden chamber assay was performed as described previously (Neuroprobe, Cabin John, MD, USA) (18). Cells were trypsinized and resuspended in 10\% DMEM. DMEM (800 $\mu \mathrm{l} /$ well; $10 \%)$ containing various concentrations of apelin-13, compound C and LY294002 was added into the wells in the lower chamber and $1.5 \times 10^{4}$ cells (200 $\mu \mathrm{l} /$ well) were added in the upper chamber. The chambers were incubated for $8-12 \mathrm{~h}$ at $37^{\circ} \mathrm{C}$ in a $5 \% \mathrm{CO}_{2}$ humidified incubator. The cells migrating through the filter (cells on the lower side of the filter) were fixed in $4 \%$ paraformaldehyde for 10 min, stained with $0.1 \%$ crystal violet stain solution (Sigma, St. Louis, MO, USA) for $30 \mathrm{~min}$ and then five random microscopic fields per well were quantified. Each experiment was performed twice in triplicate.

Tube formation assay. The formation of vascular-like structures by MMVECs on growth factor-reduced Matrigel (BD Biosciences, Franklin Lakes, NJ, USA) was performed as previously described (19) and 24-well culture plates were coated with Matrigel according to the manufacturer's instructions. The MMVECs were seeded on coated plates at 


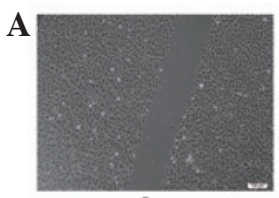

0

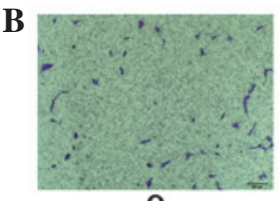

0

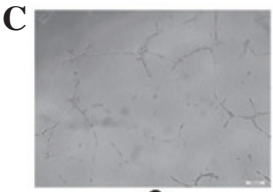

0

D

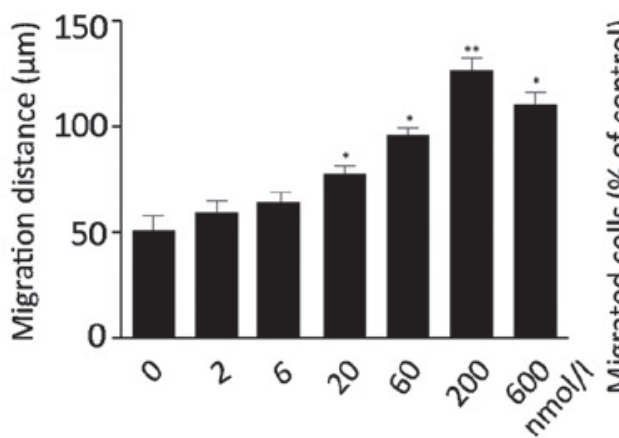

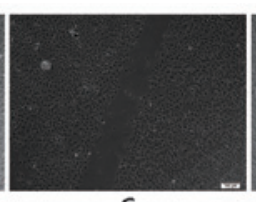

6

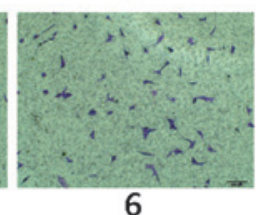

6

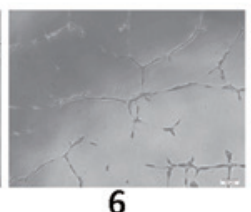

E

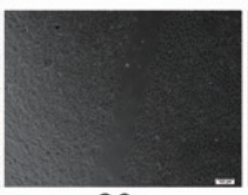

20

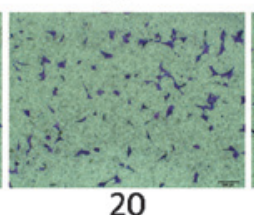

20

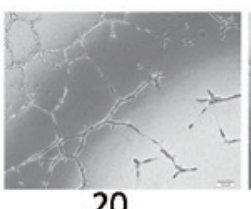

20

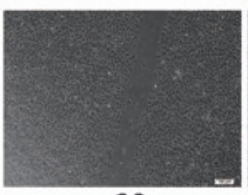

60

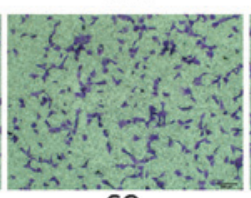

60

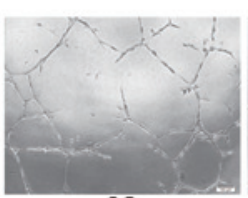

60

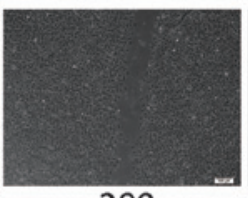

200

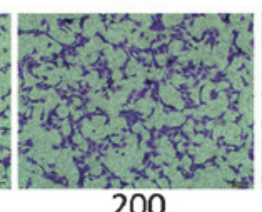

200

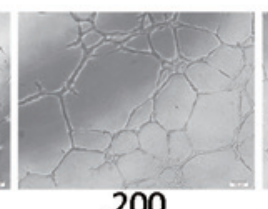

200

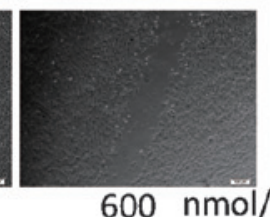

$600 \mathrm{nmol} / \mathrm{l}$

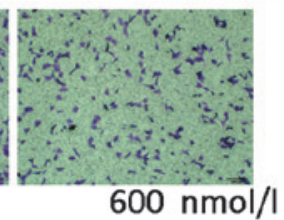

$600 \mathrm{nmol} / \mathrm{l}$

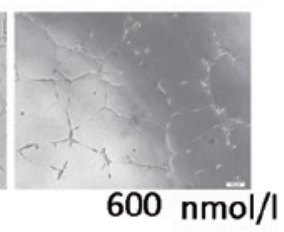

F
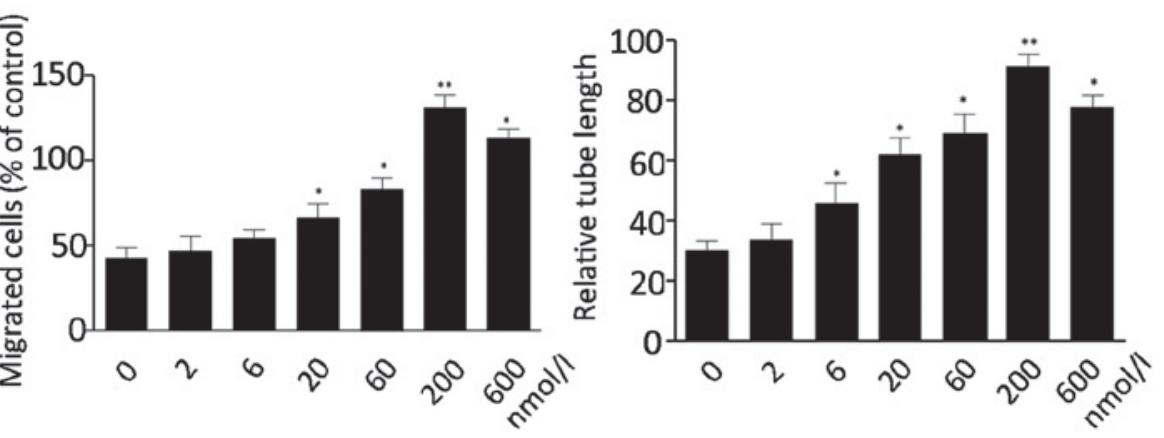

Figure 2. Apelin-13 promotes the migration of MMVECs and their differentiation into tube-like structures. (A) Migration ability of MMVECs was examined by the scratch assay. Representative images are shown (magnification, x200). (B) Representative images of the migration of MMVECs assessed by Boyden chamber analysis. (C) Representative images of in vitro tube formation analysis of MMVECs on Matrigel. (D-F) Quantitative analysis of the migration (A and B) and tube formation (C) assays, respectively. Results are expressed as the mean \pm SD. Each treatment was performed in triplicate and four fields were randomly selected from each well for counting the mean number of migrated cells. Two independent experiments were performed. Data are expressed as the mean \pm SD. Results are expressed relative to the control. ${ }^{*} \mathrm{P}<0.05$ and ${ }^{* *} \mathrm{P}<0.01$ versus the control. MMVECs, myocardial microvascular endothelial cells; SD, standard deviation.

$5 \times 10^{4}$ cells/well in DMEM containing $10 \%$ FBS and incubated at $37^{\circ} \mathrm{C}$ for $16-18 \mathrm{~h}$. Tube formation was observed using an inverted phase contrast microscope (Nikon, Tokyo, Japan). Images were captured with a video graphic system (DEI-750 CE Digital Output Camera; Optronics, Goleta, CA, USA). The degree of tube formation was quantified by measuring the length of tubes from three randomly selected low power fields (x100) from each well using the NIH Image Program. Each experiment was repeated three times.

Western blot analysis. Western blot analysis was performed as previously described (20). In brief, cell lysates were extracted with Nonidet P-40 lysis buffer followed by SDS-PAGE. The membranes were immunoblotted with the indicated primary antibodies at a 1:1,000 dilution overnight, followed by the secondary antibody conjugated with horseradish peroxidase at a 1:2,000 dilution for $2 \mathrm{~h}$. A western blotting detection kit (Amersham Biosciences, Piscataway, NJ, USA) and enhanced chemiluminescence reagent (Thermo Fisher Scientific, Waltham, MA, USA) were used to examine the membranes. Protein bands were evaluated by densitometry using Image J software (version 1.41; National Institutes of Health, Bethesda, MD, USA) and normalized to the expres- sion levels of GAPDH for protein loading. Phospho-specific signals, normalized against the quantity of total protein, are shown as arbitrary units. The changes in phosphorylation were calculated by comparing the differences between basal and stimulated values of specifically treated samples and their respective controls.

Statistical analysis. The results are expressed as the mean \pm standard deviation. Statistical comparisons were performed using analysis of variance with Scheffe's F-test for post hoc analysis. $\mathrm{P}<0.05$ was considered to indicate a statistically significant difference. Statistical analyses were performed using the SPSS 13.0 statistical software (SPSS, Chicago, IL, USA).

\section{Results}

Apelin-13 promotes the proliferation, migration and tube formation of MMVECs. The effect of apelin-13 on the proliferation of MMVECs was examined using the MTT assay. It was revealed that apelin-13 was able to promote the proliferation of MMVECs in a dose-dependent manner with a maximum effect being observed at $200 \mathrm{nmol} / \mathrm{l}$ (Fig. 1). The role of 


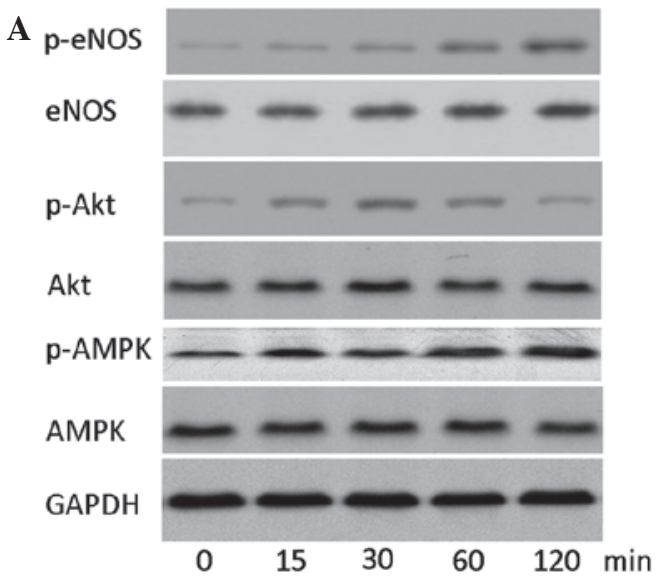

B

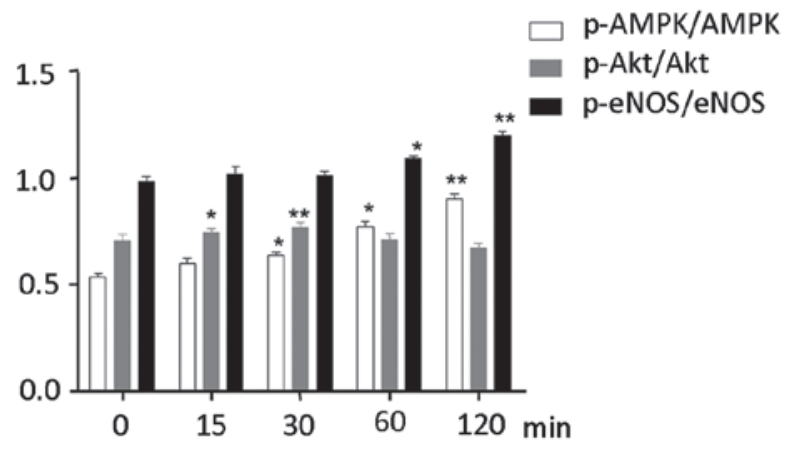

C p-eNOS

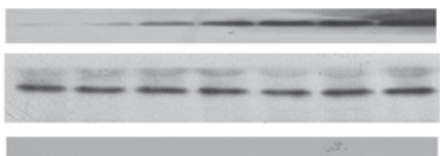

p-Akt

Akt

p-AMPK

AMPK

GAPDH
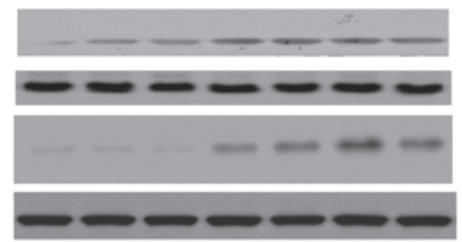

$\begin{array}{lllllll}0 & 2 & 6 & 20 & 60 & 200 & 600 \mathrm{nmol} / \mathrm{l}\end{array}$
D

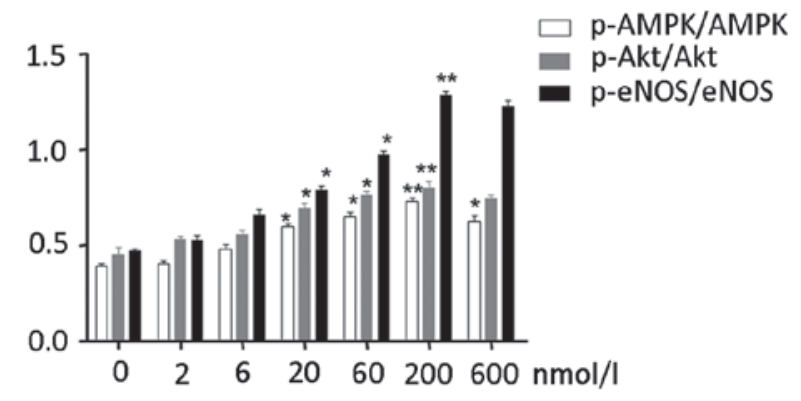

E

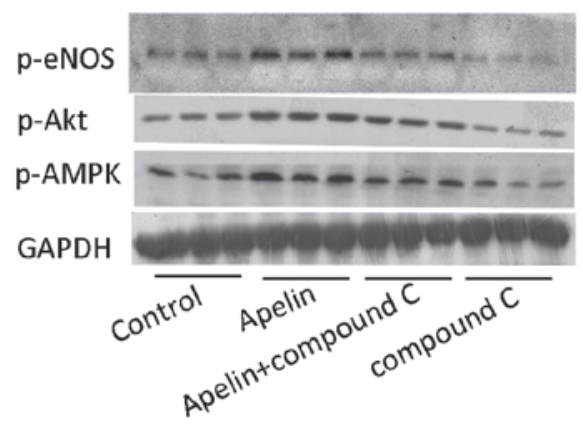

G

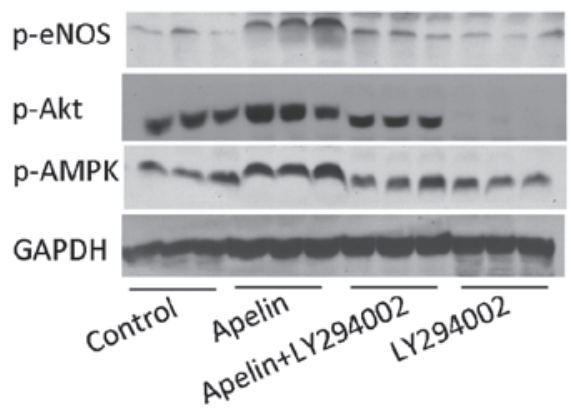

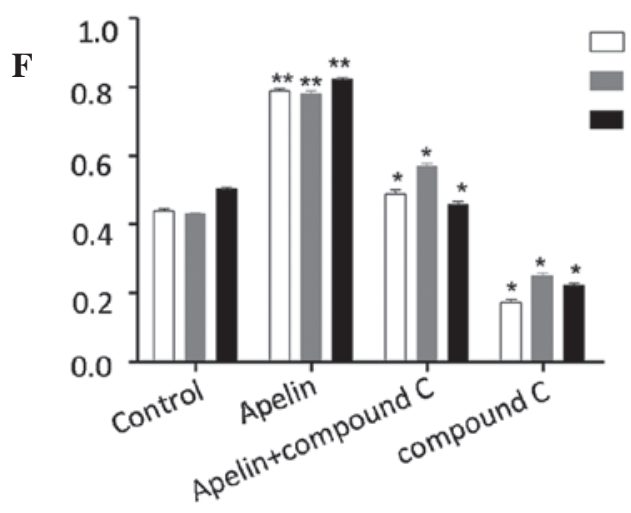

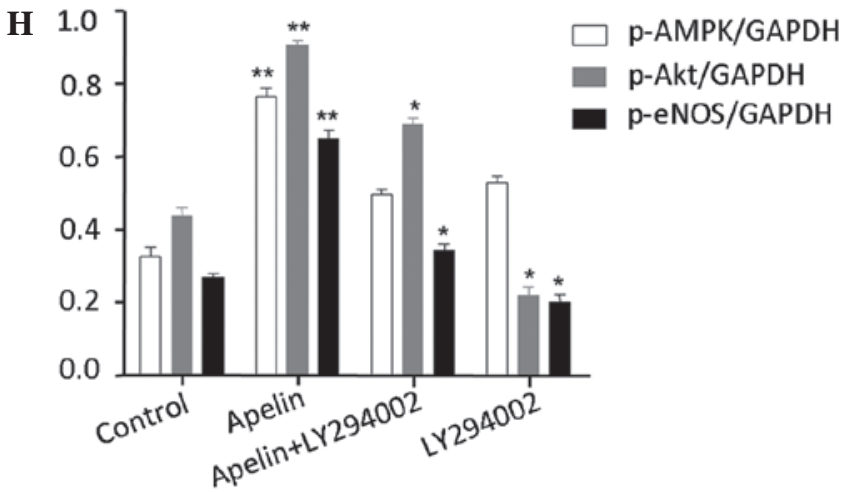

Figure 3. Expression and phosphorylation of AMPK, Akt and eNOS in MMVECs. (A) Time- (0-120 min) dependent changes in the phosphorylation of AMPK, Akt and eNOS following Aplelin-13 treatment. (C) Dose- (0-600 nmol/l) dependent changes in the phosphorylation of AMPK, Akt and eNOS following Aplelin-13 treatment. (B and D) The quantitative results are expressed relative to the total protein level. ${ }^{*} \mathrm{P}<0.05$ versus the control. (E) Role of AMPK in the regulation of apelin-13-stimulated protein phosphorylation. MMVECs were pretreated with compound C (20 $\mu \mathrm{mol} / 1)$ for 40 min, then cells were treated with apelin-13 (200 nmol/l) for $2 \mathrm{~h}$. (G) Role of Akt in the regulation of apelin-13-induced protein phosphorylation. MMVECs were pretreated with LY294002 $(20 \mu \mathrm{mol} / \mathrm{l})$ for $40 \mathrm{~min}$, cells were then treated with apelin-13 (200 nmol/l) for $2 \mathrm{~h}$. ( $\mathrm{F}$ and $\mathrm{H})$ the quantitative results are shown. ${ }^{*} \mathrm{P}<0.05$ and ${ }^{* * *} \mathrm{P}<0.01$ versus the control. AMPK, AMP-activated protein kinase; eNOS, endothelial nitric oxide synthase; Akt, protein kinase Akt/protein kinase B; MMVECs, myocardial microvascular endothelial cells. 


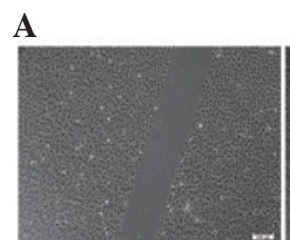

Control

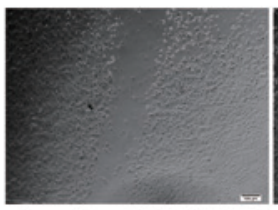

Apelin+LY294002

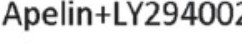

C

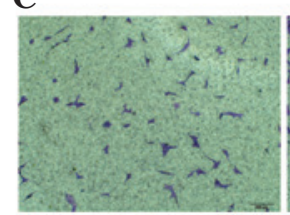

Control

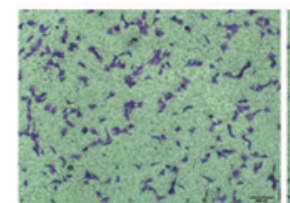

Apelin+LY294002

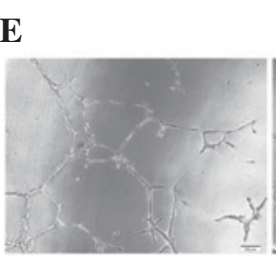

Control

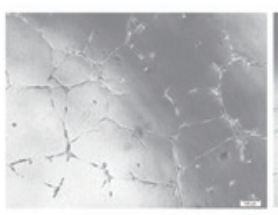

Apelin+LY294002

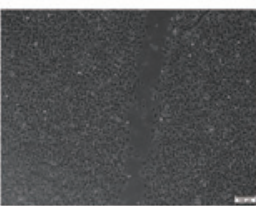

Apelin

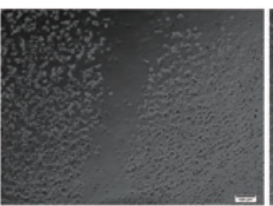

compound C

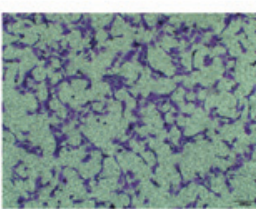

Apelin

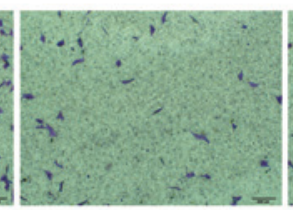

compound $\mathrm{C}$

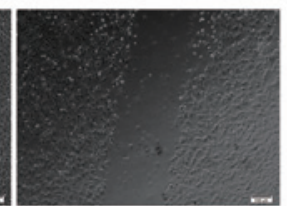

Apelin+compound C

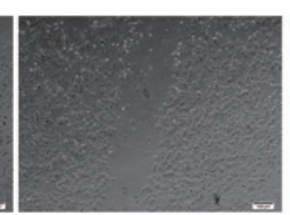

LY294002

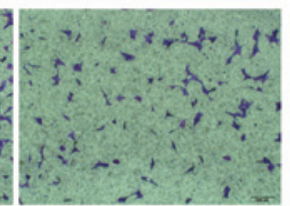

Apelin+compound C

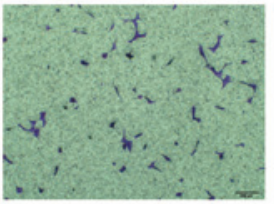

LY294002
E

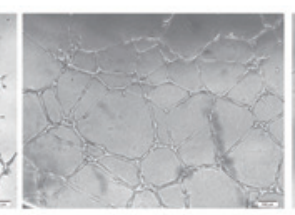

Apelin
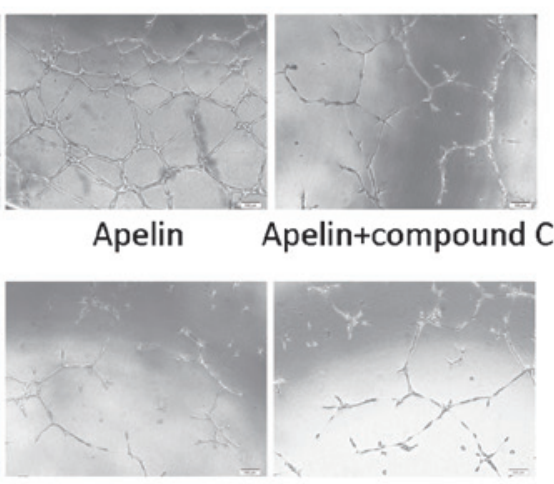

compound $\mathrm{C}$

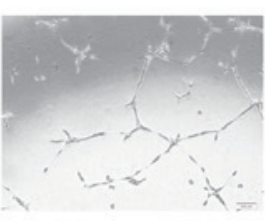

LY294002
B

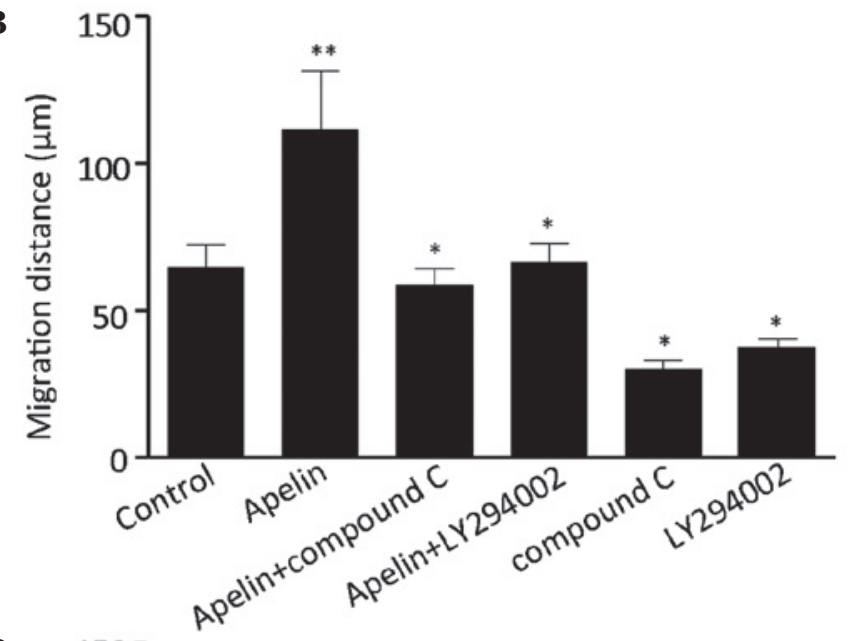

D

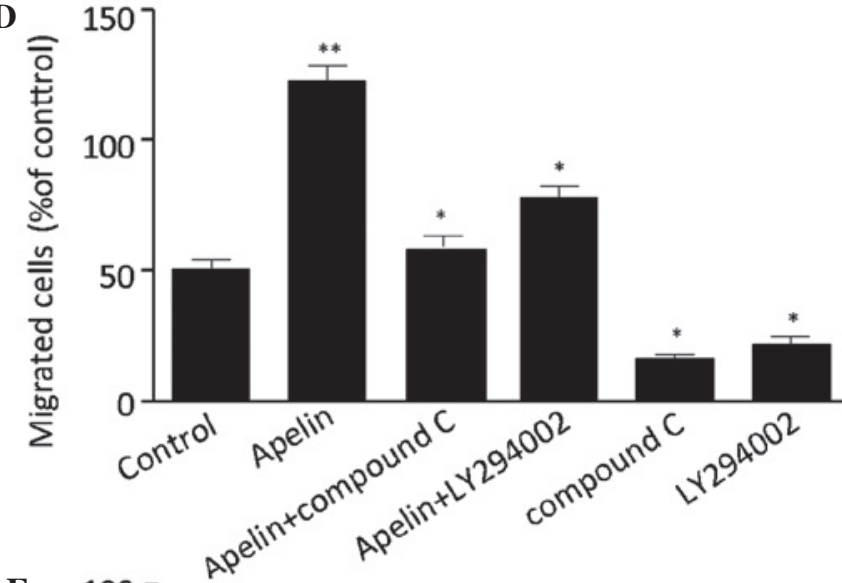

F

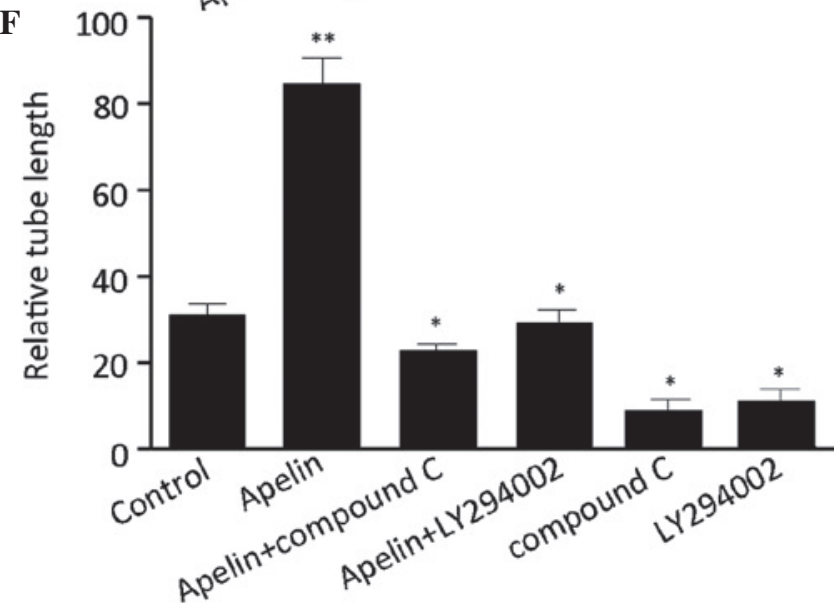

Figure 4. Roles of AMPK and Akt in Apelin-13 induced angiogenesis. MMVECs were treated with apelin-13 (200 nmol/1) or the vehicle in the presence of compound C $(20 \mu \mathrm{mol} / 1)$ or LY294002 $(20 \mu \mathrm{mol} / 1)$ at the time of seeding. (A) Scratch assay and the (C) Boyden chamber assay were performed after MMVECs were pretreated with compound C (20 $\mu \mathrm{mol} / \mathrm{l})$ or LY294002 (20 $\mu \mathrm{mol} / \mathrm{l})$. (E) Matrigel assay was used to measure the tube formation of MMVECs. (B and D) Quantitative analysis of migration distance and (F) tube length is shown as the mean \pm standard deviation. Results are expressed relative to the control. ${ }^{*} \mathrm{P}<0.05$ and ${ }^{* *} \mathrm{P}<0.01$ versus the control. AMPK, AMP-activated protein kinase; MMVECs, myocardial microvascular endothelial cells; LY294002, Akt inhibitor; compound C, AMPK inhibitor.

apelin-13 on endothelial cell migration was further evaluated by the scratch assay and the modified Boyden chamber assay. Apelin-13 significantly stimulated the migration of cells in a dose-dependent manner with an optimal activity at $200 \mathrm{nmol} / \mathrm{l}$ (Fig. 2A and B). To examine the effects of apelin-13 on the differentiation of MMVECs into vascular structures in vitro, tube formation ability was assessed. Apelin-13 significantly increased the tube formation of MMVECs in a dose-dependent manner with its optimal activity at $200 \mathrm{nmol} / \mathrm{l}$ (Fig. 2C).

Apelin-13 induces the phosphorylation of AMPK and Akt in MMVECs. It is known that AMPK and Akt are involved in the regulation of angiogenesis $(11,15)$. The effect of apelin-13 on the phosphorylation status of AMPK at Thr-172 and Akt at Ser-473 was investigated in MMVECs. The treatment of 
MMVECs with apelin-13 enhanced the phosphorylation of AMPK in a dose- and time-dependent manner with maximal AMPK phosphorylation occurring at $200 \mathrm{nmol} / \mathrm{l}$ following $2 \mathrm{~h}$ exposure. Similarly, the phosphorylation of Akt was induced by apelin-13; however, this response was transient (Fig. 3A and C). Cross-talk between AMPK and Akt was previously described to regulate the phosphorylation of eNOS $(11,21)$. Therefore, eNOS phosphorylation in MMVECs was also examined. Apelin-13 stimulated eNOS phosphorylation at Ser-1179 (p-eNOS) in a time- and dose-dependent manner, while the total protein levels (eNOS) remained unchanged. (Fig. 3A and C). Subsequently, MMVECs were treated with the AMPK and Akt inhibitors, it was revealed that they significantly suppressed the apelin-13-induced AMPK, Akt and eNOS phosphorylation in MMVECs (Fig. 3E and G), while incubation with the Akt inhibitor did not alter the endogenous phosphorylation of AMPK (Fig. 3G). Therefore, it was hypothesized that there is cross-talk between AMPK and Akt in the pro-angiogenic process induced by apelin-13.

AMPK and Akt signaling are required for apelin-13-stimulated migration and differentiation. To determine whether AMPK and Akt signaling are involved in apelin-13-stimulated endothelial cell migration and tube formation in vitro, MMVECs were treated either with compound C or LY294002, the inhibitors of AMPK and Akt, respectively. Cell scratch and Boyden chamber assays were used to assess the role of AMPK and Akt signaling on the migration ability of MMVECs induced by apelin-13. It was observed that the AMPK and Akt inhibitors significantly suppressed the apelin-13-stimulated migration of MMVECs (Fig. 4A and C). Subsequently, Matrigel assays were performed to investigate the effect of these two inhibitors on the tube formation induced by apelin-13 in MMVECs. The inhibitors significantly impaired the apelin-13-stimulated tube formation (Fig. 4E) of MMVECs. These data indicated that AMPK and Akt signaling are required for apelin-13-induced endothelial migration and differentiation in MMVECs.

\section{Discussion}

In the present study, it was demonstrated that apelin-13 promotes angiogenesis through the modulation of AMPK and Akt signaling in MMVECs. It was further investigated how AMPK and Akt signaling participates in the pro-angiogenic process and it was found that the activation of eNOS is involved in the two processes.

The regulatory role of apelin- 13 in endothelial cells has previously been investigated. It was revealed that apelin is expressed in vascular endothelial cells and participates in regulating their proliferation and regenerative angiogenesis $(22,23,24)$. Calcitonin gene-related peptide and vascular endothelial growth factor were reported to promote angiogenesis by promoting AMPK and Akt signaling activation, respectively $(25,26)$. AMPK and Akt directly phosphorylate eNOS $(17,27)$, which is important in angiogenesis. By contrast, apelin-13 can regulate glucose and lipid metabolism $(12,13)$ via activating AMPK signaling. We hypothesized that apelin-13 may promote angiogenesis through the activation of AMPK and Akt signaling in MMVECs. The results demonstrated that apelin-13 can promote angiogenesis in MMVECs. Apelin-13 stimulated the phosphorylation of AMPK and eNOS in a time- and dose-dependent manner, while the phosphorylation of Akt increased in the early stages and decreased at the later stages. AMPK and Akt inhibitors suppressed the apelin-13-induced AMPK, Akt and eNOS phosphorylation and also inhibited apelin-13-stimulated cell migration and differentiation. Notably, although the AMPK inhibitor suppressed the endogenous Akt phosphorylation, the Akt inhibitor had no effect on the endogenous phosphorylation of AMPK in MMVECs. The present data suggest that apelin-13 exerts pro-angiogenic effects in MMVECs through the modulation of AMPK and Akt signaling and the activation of eNOS, which is in accordance with previous studies $(28,29,30)$. A study by Nagata et al (11) demonstrated that AMPK did not exhibit an effect on endothelial cell migration, tube formation and NO production under normoxia, which suggests that further in vitro and in vivo studies are required to gain a greater understanding of the role of endothelial cell AMPK in angiogenesis.

Of note, in the present study, it was not determined how apelin-13 regulates AMPK and Akt signaling. In addition, although a subunit of AMPK was identified as the major target, further evaluation is required regarding the specific subunit $(\alpha 1$ or $\alpha 2)$ involved. However, it was demonstrated that the activation of AMPK and Akt signaling was associated with angiogenesis induced by apelin-13 in MMVECs. Notably, the impaired angiogenesis was associated with the inhibition of the pathways.

The imbalance between angiogenesis and cardiac hypertrophy is important in the transition from adaptive hypertrophy to pathological cardiac remodeling in heart failure. Apelin-13 has been demonstrated to exert cardiovascular protective effects $(31,32)$. The results of the present study demonstrated that apelin-13 is a pro-angiogenic factor, suggesting that the exogenous supplementation of apelin-13 may improve cardiac remodeling in patients with heart failure.

\section{Acknowledgements}

This study was supported by the Natural Science Foundation of Shanghai Science and Technology Commission (grant no. 10ZR1422900 to Professor Mingya Liu) and partly supported by the National Natural Science Foundation of China (grant no. 81070110 to Professor Meng Wei).

\section{References}

1. Tatemoto K, Hosoya M, Habata Y, et al: Isolation and characterization of a novel endogenous peptide ligand for the human APJ receptor. Biochem Biophys Res Commun 251: 471-476, 1998.

2. Kawamata Y, Habata Y, Fukusumi S, et al: Molecular properties of apelin: tissue distribution and receptor binding. Biochim Biophys Acta 1538: 162-171, 2001.

3. Kälin RE, Kretz MP, Meyer AM, et al: Paracrine and autocrine mechanisms of apelin signaling govern embryonic and tumor angiogenesis. Dev Biol 305: 599-614, 2007.

4. Hosoya M, Kawamata Y, Fukusumi S, et al: Molecular and functional characteristics of APJ. Tissue distribution of mRNA and interaction with the endogenous ligand apelin. J Biol Chem 275: 21061-21067, 2000.

5. O'Carroll AM, Selby TL, Palkovits M and Lolait SJ: Distribution of mRNA encoding B78/apj, the rat homologue of the human APJ receptor, and its endogenous ligand apelin in brain and peripheral tissues. Biochim Biophys Acta 1492: 72-80, 2000.

6. Cheng X, Cheng XS and Pang CC: Venous dilator effect of apelin, an endogenous peptide ligand for the orphan APJ receptor, in conscious rats. Eur J Pharmacol 470: 171-175, 2003. 
7. Szokodi I, Tavi P, Földes G, et al: Apelin, the novel endogenous ligand of the orphan receptor APJ, regulates cardiac contractility. Circ Res 91: 434-440, 2002.

8. Farkasfalvi K, Stagg MA, Coppen SR, et al: Direct effects of apelin on cardiomyocyte contractility and electrophysiology. Biochem Biophys Res Commun 357: 889-895, 2007.

9. Ribatti D, Vacca A, Roncali L and Dammacco F: The chick embryo chorioallantoic membrane as a model for in vivo research on angiogenesis. Int J Dev Biol 40: 1189-1197, 1996.

10. Mu J, Brozinick JT Jr, Valladares O, Bucan M and Birnbaum MJ: A role for AMP-activated protein kinase in contraction- and hypoxia-regulated glucose transport in skeletal muscle. Mol Cell 7: 1085-1094, 2001.

11. Nagata D, Mogi M and Walsh K: AMP-activated protein kinase (AMPK) signaling in endothelial cells is essential for angiogenesis in response to hypoxic stress. J Biol Chem 278: 31000-31006, 2003.

12. Zhu S, Sun F, Li W, et al: Apelin stimulates glucose uptake through the PI3K/Akt pathway and improves insulin resistance in 3T3-L1 adipocytes. Mol Cell Biochem 353: 305-313, 2011.

13. Yue $\mathrm{P}$, Jin $\mathrm{H}, \mathrm{Xu} \mathrm{S}$, et al: Apelin decreases lipolysis via G(q), G(i), and AMPK-Dependent Mechanisms. Endocrinology 152: 59-68, 2011.

14. Chandra SM, Razavi H, Kim J, et al: Disruption of the apelin-APJ system worsens hypoxia-induced pulmonary hypertension. Arterioscler Thromb Vasc Biol 31: 814-820, 2011.

15. Shiojima I and Walsh K: Role of Akt signaling in vascular homeostasis and angiogenesis. Circ Res 90: 1243-1250, 2002.

16. Morales-Ruiz M, Fulton D, Sowa G, Languino LR, Fujio Y, Walsh K and Sessa WC: Vascular endothelial growth factor-stimulated actin reorganization and migration of endothelial cells is regulated via the serine/threonine kinase Akt. Circ Res 86: 892-896, 2000.

17. Luo Z, Fujio Y, Kureishi Y, et al: Acute modulation of endothelial Akt/PKB activity alters nitric oxide-dependent vasomotor activity in vivo. J Clin Invest 106: 493-499, 2000.

18. Witzenbichler B, Kureishi Y, Luo Z, Le Roux A, Branellec D and Walsh K: Regulation of smooth muscle cell migration and integrin expression by the Gax transcription factor. J Clin Invest 104: 1469-1480, 1999

19. Kureishi Y, Luo Z, Shiojima I, et al: The HMG-CoA reductase inhibitor simvastatin activates the protein kinase Akt and promotes angiogenesis in normocholesterolemic animals. Nat Med 6: 1004-1010, 2000.

20. Nagata D, Suzuki E, Nishimatsu H, et al: Cyclin A downregulation and p21(cip1) upregulation correlate with GATA-6-induced growth arrest in glomerular mesangial cells. Circ Res 87: 699-704, 2000.
21. Kovacic S, Soltys CL, Barr AJ, Shiojima I, Walsh K and Dyck JR: Akt activity negatively regulates phosphorylation of AMP-activated protein kinase in the heart. J Biol Chem 278: 39422-39427, 2003.

22. Kleinz MJ and Davenport AP: Immunocytochemical localization of the endogenous vasoactive peptide apelin to human vascular and endocardial endothelial cells. Regul Pept 118: $119-125,2004$.

23. Eyries M, Siegfried G, Ciumas M, Montagne K, Agrapart M, Lebrin F and Soubrier F: Hypoxia-induced apelin expression regulates endothelial cell proliferation and regenerative angiogenesis. Circ Res 103: 432-440, 2008.

24. Masri B, Morin N, Cornu M, Knibiehler B and Audigier Y: Apelin (65-77) activates p70 S6 kinase and is mitogenic for umbilical endothelial cells. FASEB J 18: 1909-1911, 2004.

25. Zheng S, Li W, Xu M, et al: Calcitonin gene-related peptide promotes angiogenesis via AMP-activated protein kinase. Am J Physiol Cell Physiol 299: C1485-C1492, 2010.

26. Radisavljevic Z, Avraham H and Avraham S: Vascular endothelial growth factor up-regulates ICAM-1 expression via the phosphatidylinositol $3 \mathrm{OH}$-kinase/AKT/Nitric oxide pathway and modulates migration of brain microvascular endothelial cells. J Biol Chem 275: 20770-20774, 2000.

27. Morrow VA, Foufelle F, Connell JM, Petrie JR, Gould GW and Salt IP: Direct activation of AMP-activated protein kinase stimulates nitric-oxide synthesis in human aortic endothelial cells. J Biol Chem 278: 31629-31639, 2003.

28. Zhu Z, Fu C, Li X, et al: Prostaglandin E2 promotes endothelial differentiation from bone marrow-derived cells through AMPK activation. PLoS One 6: e23554, 2011.

29. $\mathrm{Su} \mathrm{KH}, \mathrm{Yu} \mathrm{YB}, \mathrm{Hou} \mathrm{HH}$, et al: AMP-activated protein kinase mediates erythropoietin-induced activation of endothelial nitric oxide synthase. J Cell Physiol 227: 3053-3062, 2012.

30. Izumi Y, Shiota M, Kusakabe H, et al: Pravastatin accelerates ischemia-induced angiogenesis through AMP-activated protein kinase. Hypertens Res 32: 675-679, 2009.

31. Tao J, Zhu W, Li Y, et al: Apelin-13 protects the heart against ischemia-reperfusion injury through inhibition of ER-dependent apoptotic pathways in a time-dependent fashion. Am J Physiol Heart Circ Physiol 301: H1471-H1486, 2011.

32. Rastaldo R, Cappello S, Folino A, et al: Apelin-13 limits infarct size and improves cardiac postischemic mechanical recovery only if given after ischemia. Am J Physiol Heart Circ Physiol 300: H2308-H2315, 2011 